\title{
RPL6: A Key Molecule Regulating Zinc- and Magnesium-Bound Metalloproteins of Parkinson's Disease
}

\author{
Athira Anirudhan ${ }^{1 \dagger}$, Paola Isabel Angulo-Bejarano ${ }^{2 t}$, Prabu Paramasivam ${ }^{3}$, \\ Kalaivani Manokaran ${ }^{4}$, S. Manjunath Kamath ${ }^{5}$, Ram Murugesan ${ }^{1}$, Ashutosh Sharma ${ }^{2 *}$ \\ and Shiek S. S. J. Ahmed ${ }^{1 *}$
}

\begin{abstract}
${ }^{1}$ Drug Discovery and Multi-omics Laboratory, Faculty of Allied Health Sciences, Chettinad Academy of Research and Education, Kelambakkam, India, ${ }^{2}$ School of Engineering and Sciences, Centre of Bioengineering, Tecnologico de Monterrey, Queretaro, Mexico, ${ }^{3}$ Department of Neurology, School of Medicine, University of New Mexico Health Sciences Center, University of New Mexico, Albuquerque, NM, United States, ${ }^{4}$ Department of Medical Laboratory Technology, Manipal College of Health Professions, Manipal, Manipal Academy of Higher Education, Manipal, India, ${ }^{5}$ Department of Pharmacology, Saveetha Dental College (SDC), Saveetha Institute of Medical and Technical Sciences, Chennai, India
\end{abstract}

OPEN ACCESS

Edited by:

Natalia Polouliakh,

Sony Computer Science

Laboratories, Japan

Reviewed by:

Siliang Chen,

Peking Union Medical College

Hospital (CAMS), China

Paulami Chatterjee,

Stanford University, United States

${ }^{*}$ Correspondence:

Ashutosh Sharma

asharma@tec.mx

Shiek S. S. J. Ahmed

shiekssjahmed@gmail.com

tThese authors have contributed equally to this work and share first authorship

Specialty section:

This article was submitted to

Systems Biology,

a section of the journal

Frontiers in Neuroscience

Received: 02 December 2020

Accepted: 25 January 2021

Published: 11 March 2021

Citation:

Anirudhan A, Angulo-Bejarano PI,

Paramasivam P, Manokaran $K$, Kamath MS, Murugesan R, Sharma A and Ahmed SSSJ (2021) RPL6: A Key

Molecule Regulating Zincand Magnesium-Bound

Metalloproteins of Parkinson's

Disease. Front. Neurosci. 15:631892.

doi: 10.3389/fnins.2021.631892
Parkinson's disease (PD) is a progressive neurodegenerative disease with no definite molecular markers for diagnosis. Metal exposure may alter cellular proteins that contribute to PD. Exploring the cross-talk between metal and its binding proteins in PD could reveal a new strategy for PD diagnosis. We performed a meta-analysis from different PD tissue microarray datasets to identify differentially expressed genes (DEGs) common to the blood and brain. Among common DEGs, we extracted 280 metalloprotein-encoding genes to construct protein networks describing the regulation of metalloproteins in the PD blood and brain. From the metalloprotein network, we identified three important functional hubs. Further analysis shows 60S ribosomal protein L6 (RPL6), a novel intermediary molecule connecting the three hubs of the metalloproteins network. Quantitative real-time PCR analysis showed that RPL6 was downregulated in PD peripheral blood mononuclear cell (PBMC) samples. Simultaneously, trace element analysis revealed altered serum zinc and magnesium concentrations in PD samples. The Pearson's correlation analysis shows that serum zinc and magnesium regulate the RPL6 gene expression in PBMC. Thus, metal-regulating RPL6 acts as an intermediary molecule connecting the three hubs that are functionally associated with PD. Overall our study explores the understanding of metal-mediated pathogenesis in $\mathrm{PD}$, which provides a serum metal environment regulating the cellular gene expression that may light toward metal and gene expression-based biomarkers for PD diagnosis.

Keywords: Parkinson's disease, meta-analysis, metalloprotein network, gene expression, serum metals

\section{INTRODUCTION}

Parkinson's disease (PD) is one of the most prevalent neurodegenerative diseases occurring at the substantia nigra $(\mathrm{SNc}$ ) region of the central nervous system (Lang and Lozano, 1998). Degeneration of neurons causes resting bradykinesia, postural instability, rigidity, and tremor. These symptoms, along with the Movement Disorder Society (MDS)-Unified Parkinson's Disease Rating Scale (UPDRS), help in the clinical diagnosis of 
PD (Magrinelli et al., 2016). Imaging techniques such as CT, PET, and MRI showed benefit in PD detection (Pagano et al., 2016). Currently, there is no molecular method to diagnose PD and treatment to cure PD. The medications help in symptom management. Advances in research establish the underlying PD pathogenesis that allows determining molecular markers for diagnosis. Many studies implement high-throughput technologies, which generate vast biological data in genomics, transcriptomics, proteomics, and metabolomics. These biological resources publically available in repositories are useful for further investigation. Integrating these available resources may provide several previously unknown mechanisms of $\mathrm{PD}$, which enables biomarker discovery.

Among biological data, meta-analysis of microarray-based gene expression profiles was extensively investigated to overcome the difficulties in conducting a large cohort study. Meta-analysis is a scientific method that systematically integrates the gene expression data of different populations on a similar entity to analyze and provide more reliable results (Ahn and Kang, 2018). Although several gene expression studies were conducted in $\mathrm{PD}$, the potential limitation of obtaining the affected tissue restricts them to conduct an experiment in a larger sample size. A recent review highlights the restriction of less sample size in most PD gene expression studies (Kelly et al., 2019). Hence, a meta-analysis of combining the gene expression datasets of various PD studies will increase the sample size and provide more reliable results with a high statistical power. For instance, Chi et al. (2018) executed the meta-analysis and reported that mitogen-activated protein kinase 8 (MAPK8), cell division cycle 42 (CDC42), NADH:ubiquinone oxidoreductase core subunit S1 (NDUFS1), cytochrome C oxidase subunit 4I1 (COX4I1), and succinate dehydrogenase complex subunit $\mathrm{C}(\mathrm{SDHC})$ are the potential markers for PD diagnosis. However, Chi et al. (2018) conducted a meta-analysis in brain tissue, which diminishes the light to use these markers for clinical practices. Testing the genes that are pathologically connecting brain and blood through a meta-analysis will help develop blood-based biomarkers (Moni et al., 2019; Shen et al., 2019; Genoud et al., 2020). Such pathologically linked biomarkers will be more reliable and improve the diagnostic value.

Considering various environmental factors, metal exposure is one of the most frequently reported causative factors in PD. Several reports suggest altered metal concentration in PD (Forte et al., 2004; Bocca et al., 2006). However, the influence of metal in regulating $\mathrm{PD}$ genes in the context of biological network is limited. We hypothesized that the environmental metals alter the metal homeostasis that may influence the cellular metal-binding proteins to cause PD. In order to understand PD efficiently in the context of metal toxicity, the metalloprotein network involving metal-bound proteins and metals need to be studied.

\section{MATERIALS AND METHODS}

The retrieval, selection, analysis, and reporting of this study follow the Preferred Reporting Items for Systematic Reviews and Meta-Analyses (PRISMA) guidelines (Moher et al., 2015).

\section{Dataset Selection for Meta-Analysis}

Gene expression dataset searches were executed in NCBI Gene Expression Omnibus $(\mathrm{GEO})^{1}$ and Array Express database ${ }^{2}$ by inception of database records between January 1990 and November 2018 using the keywords related to "Parkinson's disease." Two independent groups (SSJ, AS, and RM; PIA, $\mathrm{PP}$, and $\mathrm{MK}$ ) tested the procedure (Figure 1) of dataset search and retrieval following the inclusion and exclusion criteria as described. Inclusion criteria: (1) case-control study (Homo sapiens), (2) comparative studies between $\mathrm{PD}$ and other neurological diseases, (3) brain or whole blood as study specimens, (4) datasets include raw or processed data. Whereas the exclusion criteria include (1) animal model and in vivo studies, (2) secondary gene expression studies, (3) microarray dataset with less than two replicate samples in a group, and (4) studies other than microarray experiments.

\section{Data Extraction}

Data extraction was performed independently by two authors (AA and $\mathrm{KM}$ ) and cross-verified between them to eliminate errors. Any differences in opinion were resolved through group discussion. The information relating to each selected dataset, such as investigator name, year, accession number, sample source, array platform, number of samples (PD and control), expression data, study population, and primary and secondary outcome measures, was recorded. The characteristics of the dataset are included in Tables 1A,B.

\section{Analysis of Individual Dataset}

Each dataset was background corrected and normalized using Robust Multi-chip Averaging (RMA) method. Principal component analysis (PCA) plot was visualized to determine the sample distribution based on gene expression before and after normalization to identify outliers. The differentially expressed genes (DEGs) in PD compared to control was computed using the limma method (Derkow et al., 2018) with $p$-value $<0.05$, adjusted by Benjamini-Hochberg's false discovery rate (FDR).

\section{Meta-Analysis}

Meta-analysis was performed using MetaDE package in $\mathrm{R}$ (Wang et al., 2012) by following the procedure of Su et al. (2019). Datasets from the same tissue (blood or brain) were combined to perform the meta-analysis. The RMA processed expression datasets were then merged by converting probe ID of each gene to Entrez ID, which allows comparing the same gene across different datasets. Further, the homogeneity of all the collected datasets was verified using MetaDE package in R (Wang et al., 2012). Further, Fisher's method was used with $p$-value $<0.05$ from the multiple datasets to determine the significantly differentially expressed meta-analyzed PD genes of the brain and blood.

\footnotetext{
${ }^{1}$ http://www.ncbi.nlm.nih.gov/geo/

${ }^{2}$ http://www.ebi.ac.uk/arrayexpress/
} 


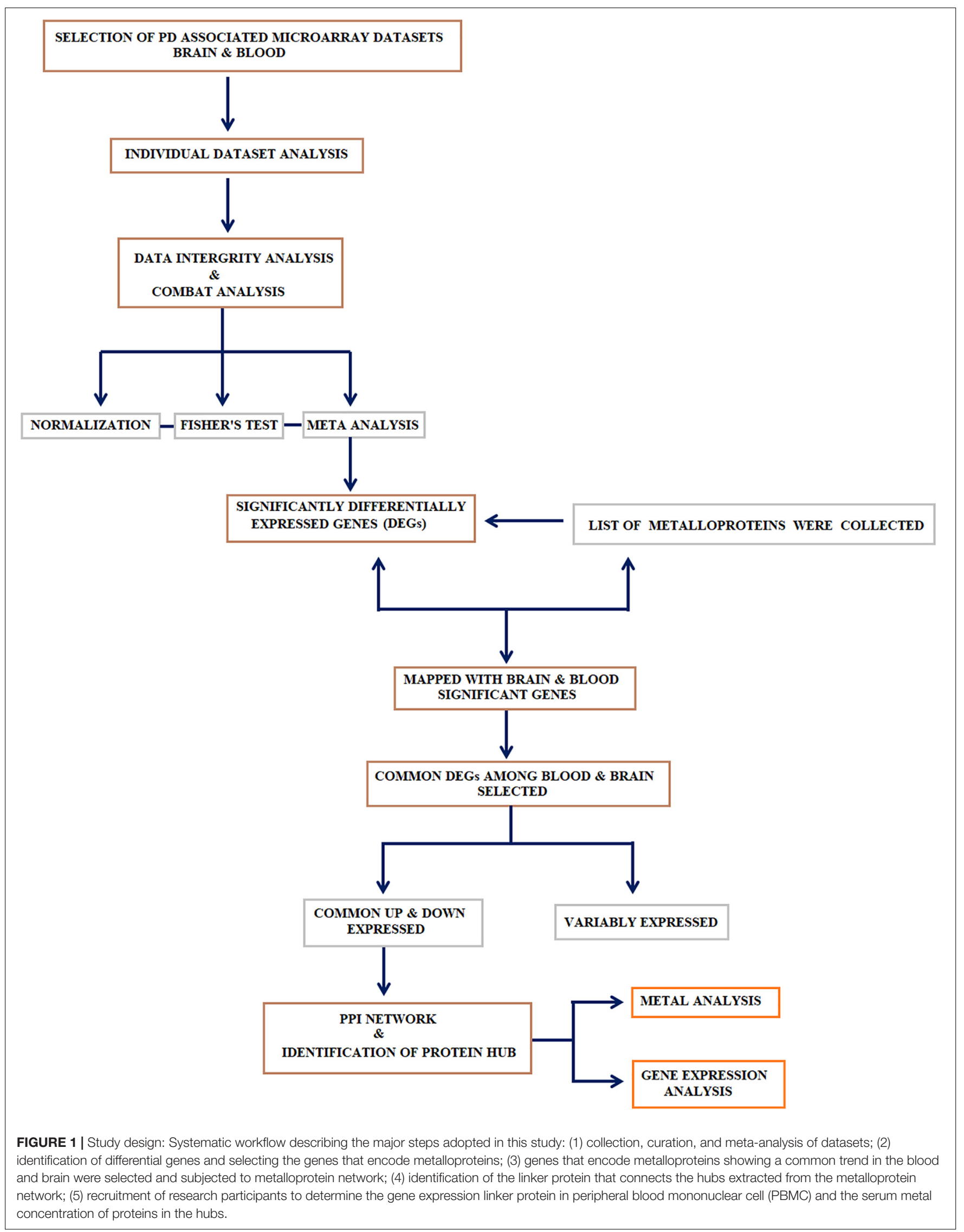


TABLE 1A | The study characteristics of brain datasets included in the meta-analysis.

\begin{tabular}{|c|c|c|c|}
\hline \multirow[t]{2}{*}{ Accession ID } & \multirow[t]{2}{*}{ Array platform number } & \multicolumn{2}{|c|}{ Number of samples } \\
\hline & & Control & PD \\
\hline GSE7621 & GPL570 [HG-U133_Plus_2] & 9 & 16 \\
\hline \multirow[t]{2}{*}{ GSE8397 } & GPL96 [HG-U133A] & 19 & 28 \\
\hline & GPL97 [HG-U133B] & 17 & 29 \\
\hline GSE19587 & GPL571 [HG-U133A_2] & 10 & 12 \\
\hline GSE20141 & GPL570 [HG-U133_Plus_2] & 8 & 10 \\
\hline GSE20146 & GPL570 [HG-U133_Plus_2] & 10 & 10 \\
\hline GSE20163 & GPL96 [HG-U133A] & 9 & 8 \\
\hline GSE20164 & GPL96 [HG-U133A] & 5 & 6 \\
\hline GSE20168 & GPL96 [HG-U133A] & 15 & 14 \\
\hline \multirow[t]{2}{*}{ GSE20186 } & GPL96 [HG-U133A] & 14 & 14 \\
\hline & GPL6947 Illumina HumanHT-12 V3.0 expression beadchip & 17 & 16 \\
\hline GSE20291 & GPL96 [HG-U133A] & 20 & 15 \\
\hline GSE20292 & GPL96 [HG-U133A] & 18 & 11 \\
\hline GSE20295 & GPL96 [HG-U133A] & 53 & 40 \\
\hline GSE28894 & GPL6104 Illumina humanRef-8 v2.0 expression beadchip & 59 & 55 \\
\hline GSE49036 & GPL6104 Illumina humanRef-8 v2.0 expression beadchip & 8 & 20 \\
\hline GSE42966 & GPL4133 Agilent-014850 Whole Human Genome Microarray 4 × 44K G4112F & 6 & 9 \\
\hline
\end{tabular}

PD, Parkinson's disease.

TABLE 1B | The study characteristics of blood datasets included in the meta-analysis.

\begin{tabular}{|c|c|c|c|}
\hline \multirow[t]{2}{*}{ Accession ID } & \multirow[t]{2}{*}{ Array platform number } & \multicolumn{2}{|c|}{ Number of samples } \\
\hline & & Control & PD \\
\hline GSE6613 & GPL96 [HG-U133A] & 22 & 50 \\
\hline GSE22491 & GPL6480 Agilent-014850 Whole Human Genome Microarray 4 × 44K G4112F & 8 & 10 \\
\hline GSE54536 & GPL10558 Illumina HumanHT-12 V4.0 expression beadchip & 4 & 4 \\
\hline GSE72267 & GPL571 [HG-U133A_2] & 19 & 40 \\
\hline GSE18838 & GPL5175 [HuEx-1_0-st] Affymetrix Human Exon 1.0 ST Array [transcript (gene) version] & 12 & 18 \\
\hline GSE49126 & GPL4133 Agilent-014850 Whole Human Genome Microarray 4 × 44K G4112F & 20 & 30 \\
\hline GSE57475 & GPL6947 Illumina HumanHT-12 V3.0 expression beadchip & 49 & 93 \\
\hline GSE99039 & GPL570 [HG-U133_Plus_2] & 234 & 239 \\
\hline
\end{tabular}

\section{Metalloproteins and Differential Expression}

Next, the metal-bound protein (93 metals of the periodic table) search was executed in Protein Data Bank (PDB) by applying the search filters that include: (1) Organism type: Homo sapiens; (2) Experimental method: XRD; (3) Molecule type: Protein; (4) Sequence feature: Wildtype protein; and (5) X-ray resolution: $>3 \mathrm{~A}^{\circ}$. Overall, we retrieved 6,367 proteins that bound with 60 different metals. Each metalloprotein was named using its official gene symbol. Among 6,367 metalloprotein-encoding genes, we only took DEGs that are commonly overexpressed and downregulated in both blood and brain of PD. This exercise yielded 280 DEGs, which served input for metalloprotein network construction.

\section{Metalloprotein Network Construction, Cluster Identification, and Pathway Enrichment}

Cytoscape v3.1 is a network biology software that enables to construct and investigate the complex interaction of genes and proteins using a BisoGene plug-in. The BisoGene retrieves protein-protein interaction (PPI) data from the databases DIP, BIOGRID, HPRD, BIND, MINT, and INTACT to create the network (metalloprotein network). The 280 metalloproteins were given as input to create interaction networks connecting each other's edges. Among 280 metalloproteins, 90 forms the protein network. Whereas the other proteins were noticed to have a maximum of one interacting partner and few were without interaction. The constructed network was visualized using Cytoscape network visualizer, which shows 
TABLE 2 | Clinical characteristics and metal concentrations in serum determined by AAS showing significant changes in magnesium and zinc in PD compared with control.

\begin{tabular}{lccc}
\hline Metals & Control $(\boldsymbol{n}=\mathbf{3 2})$ & PD $(\boldsymbol{n}=\mathbf{3 3})$ & $\boldsymbol{p}$-value \\
\hline Age (years) & $65.21 \pm 5.60$ & $66.21 \pm 5.41$ & 0.48 \\
Gender & Male: 15 ; Female: 7 & Male: 14 ; Female: 9 & 0.84 \\
Age at onset (years) & - & $63.84 \pm 5.4$ & - \\
UPDRS I-Mentation, & - & $2.93 \pm 2.10$ & - \\
Behavior, and Mood & - & & - \\
UPDRS II-Activities of Daily & & $11.34 \pm 7.31$ & - \\
Living (ADL) & - & & - \\
UPDRS III-Motor & & & \\
Examination & & & \\
Serum ( $\boldsymbol{\mu}$ g/L) & $4.31 \pm 0.041$ & $5.316 \pm 0.039$ & $\leq 0.001^{*}$ \\
Magnesium & $2.846 \pm 0.046$ & $2.631 \pm 0.039$ & $\leq 0.001^{*}$ \\
Zinc & & & \\
\end{tabular}

${ }^{*} p<0.05$, statistical significance; mean $\pm S D$. AAS, atomic absorption spectrophotometer; PD, Parkinson's disease; UPDRS, Unified Parkinson's Disease Rating Scale.

the interconnectivity among dysregulating metalloproteins. Additionally, the topological characteristics of the network were tested using the network analyzer plug-in of Cytoscape. From the network, we extracted the hubs that are highly connected using the Clustering with Overlapping Neighborhood Expansion (ClusterONE) algorithm (Nepusz et al., 2012). ClusterONE detects the hub from overlapping protein complexes of the PPI data and ranked them based on $p$-value. The top three hubs showing the highest significant $p$-values were selected and subjected to Kyoto Encyclopedia of Genes and Genomes (KEGG) database for pathway analysis. Simultaneously, the hub proteins and their connections were investigated to identify any intermediary molecules connecting the three hubs. The gene expression of intermediary molecule was analyzed in PD. Also, the concentrations of the metals that bound to the hub proteins were examined in PD serum compared to healthy control.

\section{Experimental Validation in Parkinson's Disease Samples}

We obtained samples from PD patients $(\mathrm{PD}=33)$ who underwent clinical examination and MDS-UPDRS grading by a movement disorder specialist at the Chettinad Hospital and Research Institute, Tamil Nadu, India (Table 2). Similarly, the participants following the below criteria served as the control sample (control $=32$ ). Our inclusion criteria were: (1) aged group between 55 and 75 years and (2) body mass index (BMI) of $18-25 \mathrm{~kg} / \mathrm{m}^{2}$. Whereas the exclusion criteria include (1) previous history of alcohol abuse; (2) smoker or tobacco user; (3) any severe systemic diseases; (4) participants under minerals and chelating agents as supplements; (5) participants who underwent any surgery in the last 8 months; and (6) presence of any secondary parkinsonism due to accident, trauma, or drugs. Before sample collection, the signed informed consent was obtained from all the participants. Peripheral blood $6 \mathrm{ml}$ $(3 \mathrm{ml}+3 \mathrm{ml})$ was collected in the Vacutainer tube (BD, United States). Then, $3 \mathrm{ml}$ blood was centrifuged immediately for $10 \mathrm{~min}$ at $3,500 \mathrm{rpm}$, and we stored serum at $-80^{\circ} \mathrm{C}$ for metal analysis. We used the remaining $3 \mathrm{ml}$ for the isolation of

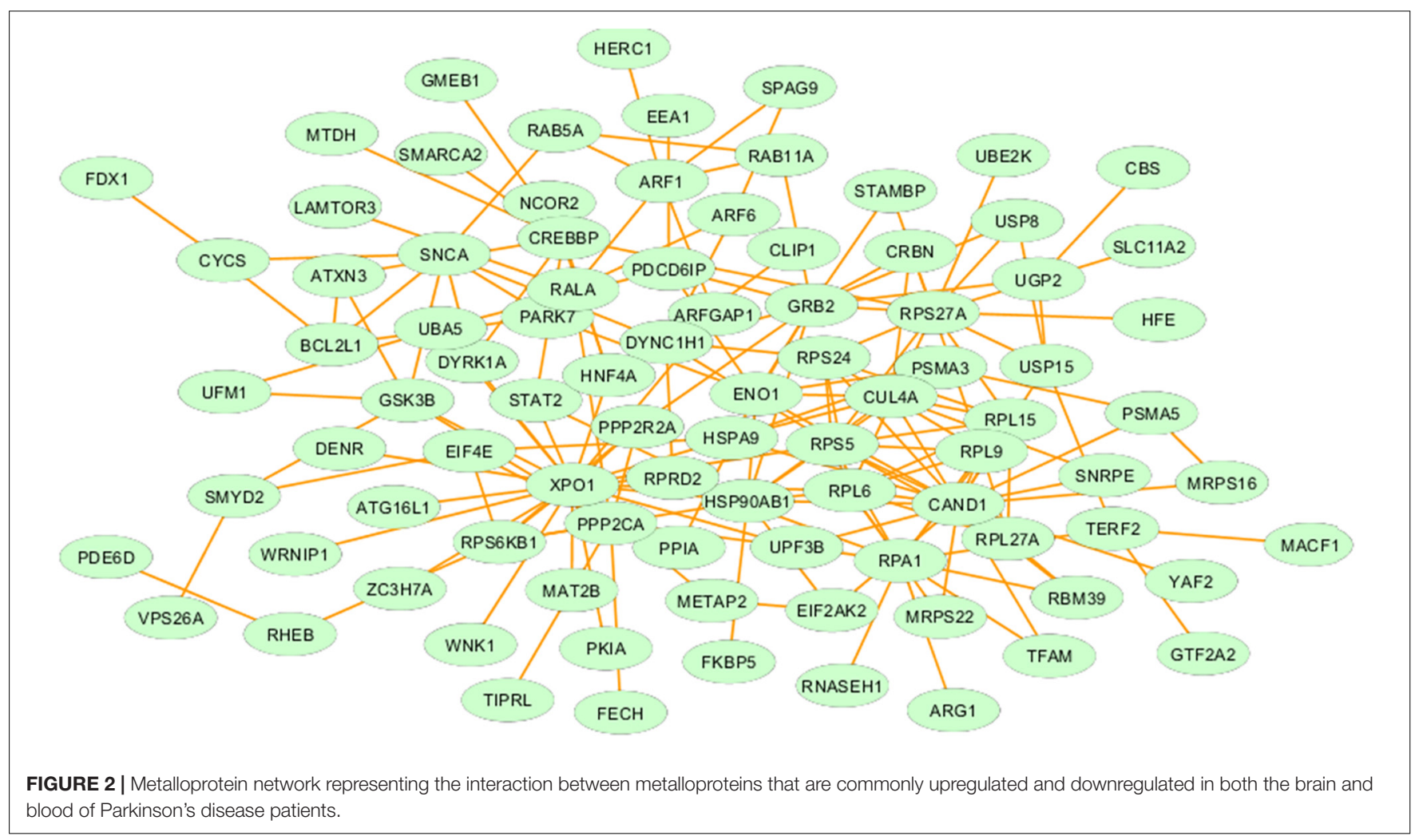



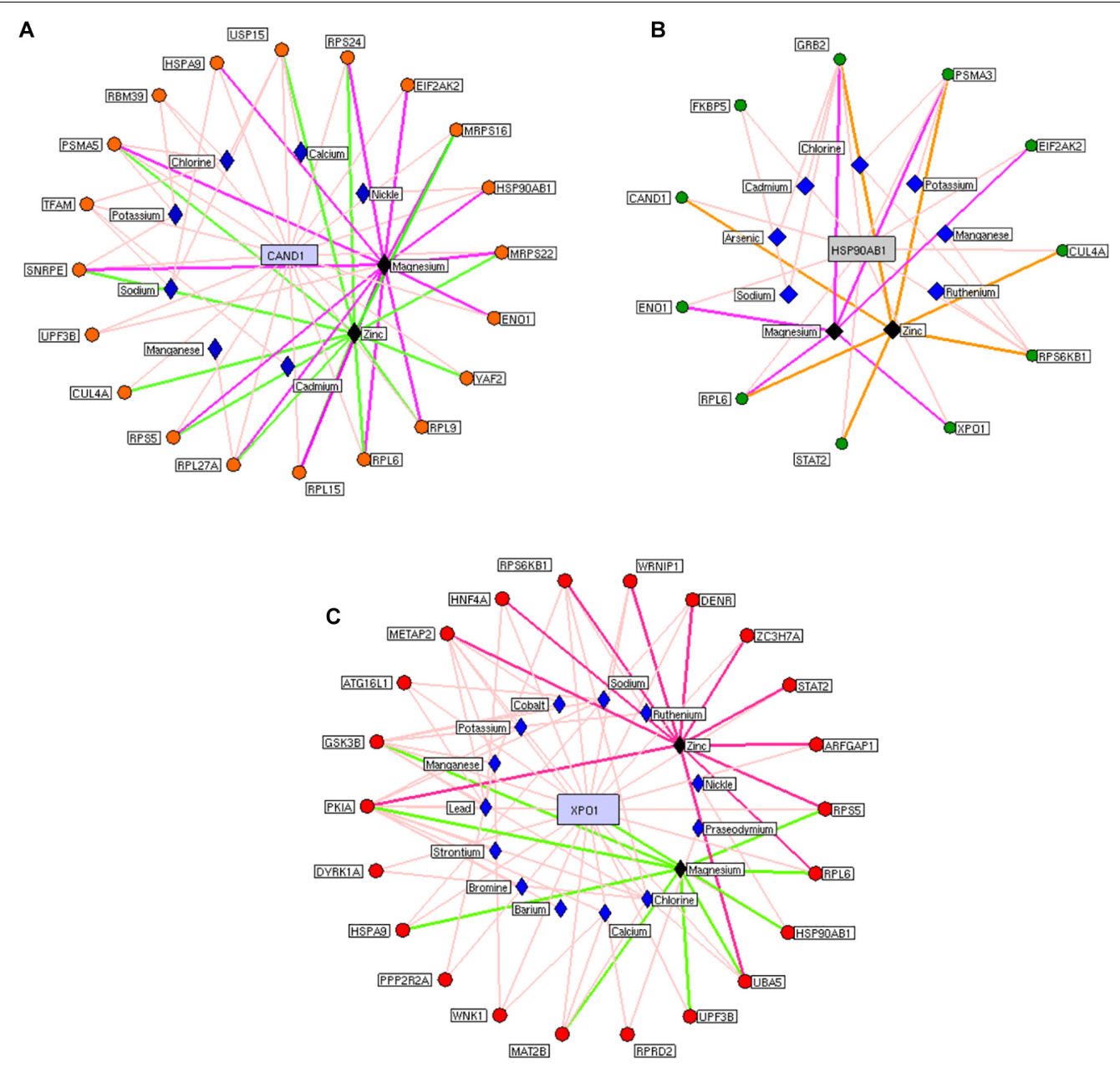

FIGURE 3 | Highly interactive metalloprotein hubs: Three highly interconnected protein hubs (A-C) extracted from the metalloprotein network. Colored circular nodes represent metalloproteins connecting to the core protein (blue rectangular box). The edges (colored line) between the proteins and their cofactor metals (violet rhombus). Black rhombus representing the cofactors that bound with more metalloproteins in the hubs.

peripheral blood mononuclear cell (PBMC) and RNA extraction for the gene expression analysis.

\section{Serum Metal Analysis}

All precautions were made by following the National Committee for Clinical Laboratory Standards (NCCLS) guidelines to avoid contamination throughout the sample processing of metal estimation. The nitric acid-based microwave digestion was carried out to extract the metals from the serum. Flame atomic absorption spectrophotometer (AAS) (Tokiya and Toda, 1977) was used to determine the serum zinc $(\mathrm{Zn})$ and magnesium $(\mathrm{Mg})$ concentration. For the calibration, NIST SRM 3100 series singleelement standard solution was used at various concentrations to have a standard graph. Also, the blank solution was used to determine the limits of detection (LoDs) for the $\mathrm{Zn}$ and $\mathrm{Mg}$.

\section{Gene Expression Analysis}

PBMCs were isolated using Histopaque-1077 (SigmaAldrich) following the manufacturer's protocol. RNA was extracted from the PBMC using TRIzol (Invitrogen) reagent and quantified using Nanodrop 2000 (Thermo Fisher Scientific). The $60 \mathrm{~S}$ ribosomal protein L6 (RPL6) gene expression was examined in $\mathrm{PD}$ and control using quantitative real-time PCR (ABI-7000, Applied Biosystems) using forward (GACGGGAATGAGAAAGGCCA) and reverse (AGCCCTGGAGCTGAGGAATA) primers. We used the gene GAPDH as internal control (forward: AAGGTGAAGGTCGGAGTCAA and reverse: ACATGTAAACCATGTAGTTGAGGT). Finally, the relative expression was measured following the $2^{-\Delta \Delta C t}$ method.

\section{Statistical Analysis}

All data were tested for normal distribution. Further, the statistical significance $(p<0.05)$ between PD and control was assessed using the Student's $t$-test method. The interdependency between metal concentration and RPL6 gene expression was calculated by Pearson's correlation method. All statistical analyses were carried using the SPSS version 21 software. 


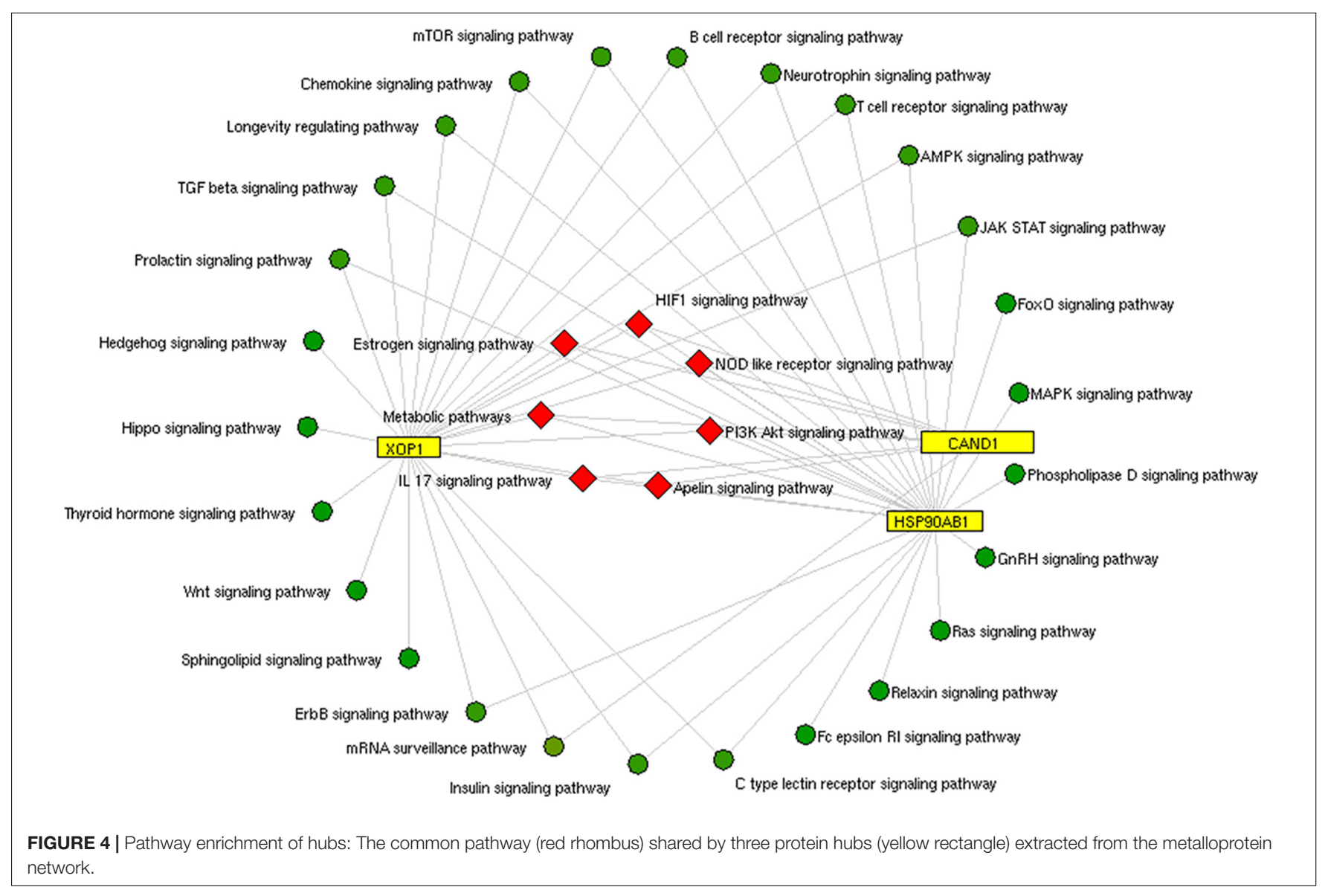

\section{RESULTS}

\section{Dataset Collection}

We used keywords related to PD to search for the gene expression studies conducted in the brain and blood in the databases. The datasets following our inclusion and exclusion criteria were selected to have 15 datasets from the brain (GSE7621, GSE8397, GSE19587, GSE20141, GSE20146, GSE20163, GSE20164, GSE20168, GSE20186, GSE20291, GSE20292, GSE20295, GSE28894, GSE49036, and GSE42966) containing 610 samples (control $=297$ and $\mathrm{PD}=313$ ) and eight datasets from the blood tissue (GSE6613, GSE22491, GSE54536, GSE72267, GSE18838, GSE49126, GSE57475, and GSE99039) containing 852 samples (control $=368, \mathrm{PD}=484$ ). The detailed characteristics of the datasets included in this study are presented in Tables 1A,B.

\section{Meta-Analysis of the Blood and Brain Datasets}

Prior to the meta-analysis, each dataset was RMA processed and DEGs between PD and control were determined using the limma package. To identify common DEGs in the blood and brain between PD and control samples, the datasets from the blood and brain were meta-analyzed with the $p<0.05$ as cutoff in Fisher's exact test. Overall, 1,951 DEGs in the blood and 3,984
DEGs in the brain were obtained. Simultaneously, 3,453 metalbound proteins were collected from the PDB, as described in the "Materials and Methods" section. We converted each metalbound protein into the official gene symbol and mapped with 1,951 (blood) and 3,984 (brain) differentially expressed metaanalyzed PD genes. A total of 280 DEGs were identified in PD showing common trends in both tissues. These 280 DEGs were used for the network construction.

\section{Metalloprotein Network and Pathway Analysis}

We constructed the metalloprotein network using Cytoscape v3.10. Among 280 metalloproteins, 90 forms the protein network that explicates 177 interconnected edges (Figure 2). Whereas the other proteins were noticed with a maximum of one interacting partner and few were without an interaction partner. The topological characteristics were assessed for the network (Supplementary Table S1). We used ClusterONE algorithm to identify the top 3 important hubs that are ranked based on p-values. Hub1 contains XPO1 with 22 interacting proteins, hub2 contains CAND1 with 20 connecting proteins, and hub3 contains HSP90AB1 connected with 11 proteins (Figure 3 and Supplementary Table S2). Each hub was subjected to pathway enrichment, which showed an association with 61 molecular pathways (XPO1 hub was associated with 26 pathways, CAND1 


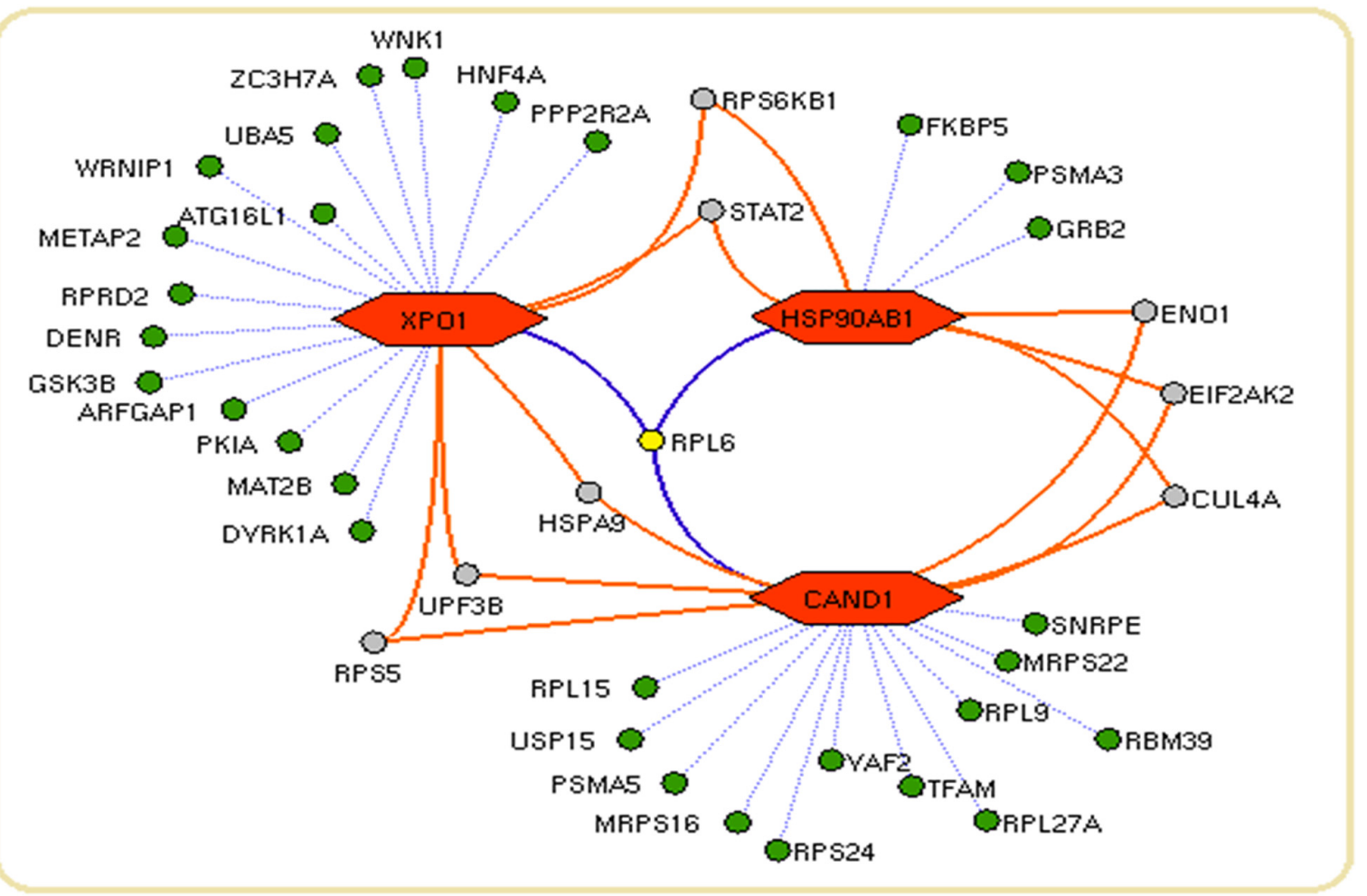

FIGURE 5 | Network connectivity of hub proteins: The network showing the proteins (green nodes) with their connected interaction (dotted violet edges). The 60S ribosomal protein L6 (RPL6) (yellow node) with violet edges that connects core proteins (orange hexagon) of the hubs. The protein (gray node) connects a maximum of two core proteins (orange hexagon) in the network.

hub linked with eight pathways, and HSP90AB1 hub was related to 27 pathways) using the KEGG database. Among 61 molecular pathways, seven were noticed common to all three hubs (Figure 4). Further, investigation using Cytoscape network analyst plug-in shows involvement of 42 proteins in the hubs, and few were common among them. Particularly, RPL6 acts as a key linker protein that connects all the three hubs (Figure 5). Subsequently, metal cofactor for the proteins in the hubs was identified (Figure 3), which shows that $\mathrm{Mg}$ and $\mathrm{Zn}$ are the cofactors for most of the metalloproteins in the hubs. Interestingly, $\mathrm{Mg}$ and $\mathrm{Zn}$ were noticed bound with RPL6 proteins (PDB ID: 6T59).

\section{Serum Metal and Peripheral Blood Mononuclear Cell Gene Expression}

The levels of $\mathrm{Mg}$ and $\mathrm{Zn}$ were assessed in the blood serum of PD and control using AAS. The normality test was conducted, which showed normal distribution of data. Further, Student's test was executed that demonstrated that Mg was significantly increased, whereas $\mathrm{Zn}$ was significantly decreased (Table 2) in PD compared to control. Similarly, the gene expression of RPL6 was decreased significantly $(p<0.05)$ in PD (Figure 6). Furthermore, the interdependency between metal concentrations and gene expression shows RPL6 was negatively correlated with $\mathrm{Mg}$ and positively correlated with $\mathrm{Zn}$ (Table 3) based on $\mathrm{R}>0.90$.

\section{DISCUSSION}

We performed a meta-analysis to identify common DEGs in PD brain and blood that allows new biological insights in PD pathogenesis. To our knowledge, this is one of the largest gene expression meta-analyses conducted in PD using 15 microarray gene expression datasets of the brain containing 610 samples and eight datasets in whole blood containing 852 samples to overcome the limitation expressed by Kelly et al. (2019). Currently, there is no well-defined molecular marker available for PD diagnosis; still the MDS-UPDRS, a gold standard method, is followed in clinical setup. A recent advancement in multi-omics technologies enables to identify biomarkers in body fluids for complex diseases (Olivier et al., 2019).

In the past decades, progression in $\mathrm{PD}$ research has been shown to be exponentially productive; however, identifying the influence of cellular metal on causative genes in PD pathogenesis is needed. Therefore, we attempted to link the serum metal concentration with the DEGs of PD. We identified DEGs by meta-analysis by merging multiple PD-associated gene expression datasets. This resulted in 280 DEGs from a protein network containing 90 proteins with 177 edges contributing to three important PD hubs representing molecular pathways.

Insulin signaling pathway is one of the molecular pathways represented by the hubs (Figure 4). Several studies reported the impaired insulin signaling as a risk factor for PD 


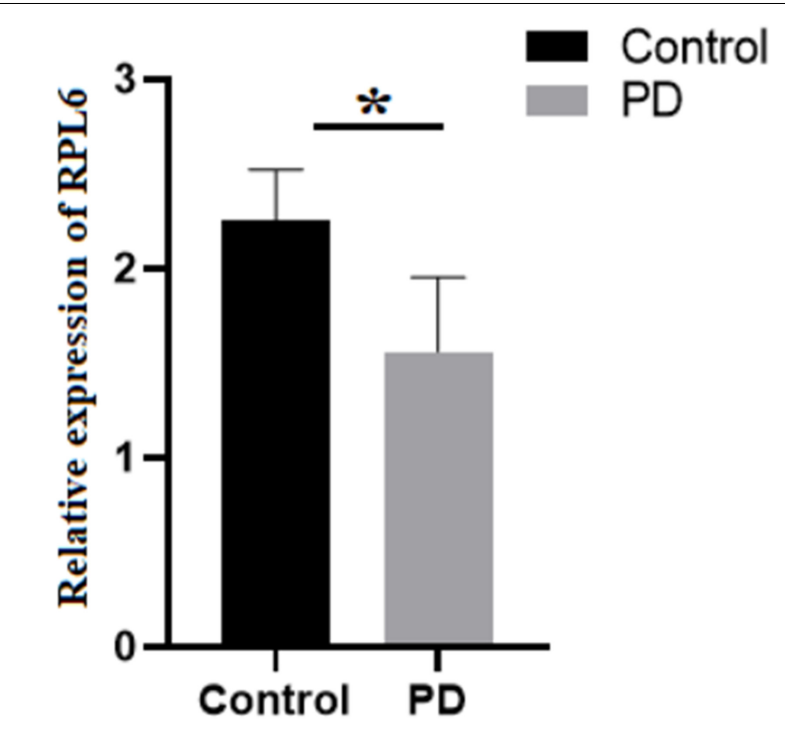

FIGURE 6 | Relative expression: Gene expression analysis of 605 ribosomal protein L6 (RPL6) in peripheral blood mononuclear cells (PBMCs) of control (black) and PD (gray). The columns represent the average gene expression with standard deviation error bar, and asterisk denotes statistical significance with $p$-value $\leq 0.05$.

(Bosco et al., 2012; Pang et al., 2016). Particularly, Bosco et al. (2012) observed a change in insulin mechanism in the cortex and the limbic PD system. Recently, Fiory et al. (2019) showed an altered blood-brain barrier (BBB) integrity in the striatum region that causes a change in the insulin signaling mechanism. Also, Khang et al. (2015) show that the insulin resistance dysregulates mitochondrial proteins at the substantia nigra (Moreira et al., 2006; Duarte et al., 2012). The disrupted mitochondrial function generates an excess of reactive oxygen species (ROS) that causes neuronal death (Dias et al., 2013). Changes in insulin signaling and mitochondrial dysfunction accelerate degeneration of dopaminergic neurons (Kleinridders et al., 2015). Also, the analysis of hubs showed involvement of mammalian target of rapamycin (mTOR) signaling and ErbB signaling pathways in PD pathogenesis. Dysregulated mTOR signaling was reported by $\mathrm{Xu}$ et al. (2014) in PD. Interestingly, Bockaert and Marin (2015) and Dijkstra et al. (2015) illustrate the upregulation of mTOR signaling pathway that accumulates $\alpha$-synuclein in stages 1 and 2 of PD.

On the other hand, ErbB signaling plays a vital role in the central nervous system. Dysregulated ErbB signaling was noticed in our pathway analysis of hubs. In PD, Iwakura et al. (2005); Iwakura and Nawa (2013) report the downregulation of ErbB that causes degeneration of dopaminergic neurons at the midbrain region. Additionally, the ErbB takes part in controlling the glutamatergic function at hippocampus and midbrain dopaminergic neurons that are associated with cognitive and behavior functions (Ledonne et al., 2018). Recently, Ledonne and Mercuri (2020) suggest that the neuregulin (NRG)/ErbBdependent synaptic plasticity at the hippocampus contributes to the memory process. The ErbB4 activation in dopaminergic
TABLE 3 | Pearson's correlation between serum metal concentrations with the RPL6 gene expression showing interdependency in Parkinson's disease.

\begin{tabular}{lccc}
\hline Metal & Gene & R & Statistical significance (p-value) \\
\hline Magnesium & RPL6 & -0.99 & $0.02^{\star}$ \\
Zinc & & 0.99 & $0.03^{*}$ \\
\hline
\end{tabular}

${ }^{*} p<0.05$, statistical significance. RPL6, 60S ribosomal protein L6.

neurons inhibits dopamine transporter (DAT) that increases the level of extracellular dopamine. Thus, frequent activation of dopamine (D4) receptor on hippocampus CA1 pyramidal neurons alters the long-term potentiation (LTP) induction or expression (Ledonne and Mercuri, 2020). Though we identified several molecular pathways, we tried to explain the importance of our study by describing the insulin, mTOR, and ErbB signaling pathways in $\mathrm{PD}$.

Subsequent analysis of hubs showed that most hub proteins were bound with $\mathrm{Zn}$ and $\mathrm{Mg}$ (Figure 3). Also, emerging evidence suggests that these pathways may be regulated by $\mathrm{Zn}$ and $\mathrm{Mg}$. Studies show $\mathrm{Zn}$ involvement in regulating the insulin pathway to maintain the homeostasis of glucose (Fukunaka and Fujitani, 2018; Norouzi et al., 2018). Zn takes part in ErbB signaling for cellular growth and differentiation (Lund et al., 2005). Szewczyk et al. (2015) suggest that decreased Zn alters the phosphorylation of mTOR signaling that dysregulates synapse and synaptic proteins. Importantly, the altered $\mathrm{Zn}$ homeostasis causes a change in behavior, memory, learning, and emotional stability (Takeda, 2000). Meta-analysis of Ke Du et al. (2017) reports decreased circulating $\mathrm{Zn}$ in $\mathrm{PD}$ patients, which is in agreement with our result. Similarly, $\mathrm{Mg}$ plays a significant role in neuromuscular signal conduction, energy synthesis, and releasing neurotransmitters (Oyanagi et al., 2006). Significant increase in $\mathrm{Mg}$ was noticed in our study; a similar result was noticed in the meta-analysis of Jin et al. (2018). Additionally, this result was further supported by our previous study (Ahmed and Santosh, 2010). Mg is essential for maintaining glucose metabolism and insulin mechanism (Kostov, 2019). Also, Mg promotes mTOR signaling (Lu et al., 2018), which confirms metals contributing to $\mathrm{PD}$ pathogenesis. Additionally, investigation of hub proteins showed RPL6 is the key linker protein that connects all three hubs. RPL6 encodes $60 \mathrm{~S}$ ribosomal subunit that plays a vital role in oxidative phosphorylation, synaptic transmission, and neuronal signaling in PD (Hamed et al., 2018). Recently, Duarte et al. (2019) suggested that downregulation of RPL6 activates AMPK signaling in PD. Similarly, downregulation of RPL6 in PBMC was observed in PD compared to control (Figure 6). Interestingly, Hamed et al. (2018) report downregulation of RPL6 in early onset, which supports early detection of PD. Also, our result suggests that altered metal homeostasis might cause a change in RPL6 gene expression, which supports our hypothesis of metal regulating cellular gene expression causing PD. Overall, our study elucidates the complex interconnection between metals and metalloproteins in PD pathogenesis. However, there are limitations in our study: (1) we have not analyzed the expression of all interacting proteins in the hubs of the metalloprotein network, (2) the number of samples enrolled for the study is 
limited, and (3) the participants involved in this study were south Indian population. On the other hand, it is important to acknowledge the advantages of this study: (1) this is one of the largest meta-analyses conducted in PD based on brain and blood gene expression profiles, (2) this study uses a novel approach that integrates the multi-omics data to provide a wider view on pathogenesis, and (3) this study provides substantial experimental evidence for the interplay between metals and cellular gene expression in PD.

\section{CONCLUSION}

In conclusion, to our knowledge, this is the first meta-analysis that investigates the association of metalloproteins and metals in PD. Our study confirms the change in serum $\mathrm{Zn}$ and $\mathrm{Mg}$ concentration in PD compared to control. Also, the altered metals contribute change in gene expression of key linker proteins connecting $\mathrm{PD}$-associated hubs. Together, relating the gene expression with metal concentration can be used as biomarkers for PD diagnosis.

\section{DATA AVAILABILITY STATEMENT}

Publicly available datasets were analyzed in this study. This data can be found here: https://www.ncbi.nlm.nih.gov/geo/query/acc. cgi? acc $=$ GSE 8397

https://www.ncbi.nlm.nih.gov/geo/query/acc.cgi?acc = GSE19587

https://www.ncbi.nlm.nih.gov/geo/query/acc.cgi?acc = GSE20141

https://www.ncbi.nlm.nih.gov/geo/query/acc.cgi?acc = GSE20146

https://www.ncbi.nlm.nih.gov/geo/query/acc.cgi?acc $\sim=\sim$ GSE20163

https://www.ncbi.nlm.nih.gov/geo/query/acc.cgi?acc $\sim=\sim$ GSE20164

https://www.ncbi.nlm.nih.gov/geo/query/acc.cgi?acc $\sim=\sim$ GSE20168

https://www.ncbi.nlm.nih.gov/geo/query/acc.cgi?acc $\sim=\sim$ GSE20186

https://www.ncbi.nlm.nih.gov/geo/query/acc.cgi?acc $\sim=\sim$ GSE20291

https://www.ncbi.nlm.nih.gov/geo/query/acc.cgi?acc $\sim=\sim$ GSE20292

https://www.ncbi.nlm.nih.gov/geo/query/acc.cgi?acc $\sim=\sim$ GSE20295

https://www.ncbi.nlm.nih.gov/geo/query/acc.cgi?acc $\sim=\sim$ GSE28894

https://www.ncbi.nlm.nih.gov/geo/query/acc.cgi?acc $\sim=\sim$ GSE49036

https://www.ncbi.nlm.nih.gov/geo/query/acc.cgi?acc $\sim=\sim$ GSE42966

https://www.ncbi.nlm.nih.gov/geo/query/acc.cgi?acc $~=\sim ~$ GSE6613

https://www.ncbi.nlm.nih.gov/geo/query/acc.cgi?acc = GSE22491 https://www.ncbi.nlm.nih.gov/geo/query/acc.cgi?acc =

GSE54536

https://www.ncbi.nlm.nih.gov/geo/query/acc.cgi?acc =

GSE72267

https://www.ncbi.nlm.nih.gov/geo/query/acc.cgi?acc =

GSE18838

https://www.ncbi.nlm.nih.gov/geo/query/acc.cgi?acc $\sim=\sim$

GSE49126

https://www.ncbi.nlm.nih.gov/geo/query/acc.cgi?acc = GSE57475

https://www.ncbi.nlm.nih.gov/geo/query/acc.cgi?acc = GSE99039.

\section{ETHICS STATEMENT}

The studies involving human participants were reviewed and approved by the Institutional Human Ethical Committee, Chettinad Academy of Research and Education. The patients/participants provided their written informed consent to participate in this study.

\section{AUTHOR CONTRIBUTIONS}

AA and PA-B conducted the experimental work and analysis and interpretation of the data. PP supervised the gene expression analysis. SA and AS designed the study, performed part of the experiment and the analysis and interpretation of the data, and critically revised the manuscript. RM supervised the statistical analysis. SK and KM provided critical suggestion in the design of the experiment and manuscript correction. Particularly, SA, RM, $\mathrm{PP}$, and SK were involved in validating the procedure for dataset collection for meta-analysis. All authors read and approved the current version of the manuscript.

\section{FUNDING}

The research was funded by the Chettinad Academy of Research and Education (CARE).

\section{ACKNOWLEDGMENTS}

We thank Chettinad Academy of Research and Education (CARE) for providing the facility to carry out the research work.

\section{SUPPLEMENTARY MATERIAL}

The Supplementary Material for this article can be found online at: https://www.frontiersin.org/articles/10.3389/fnins.2021. 631892/full\#supplementary-material

Supplementary Table 1 | Topological characteristic of the network.

Supplementary Table 2 | Average fold change in PD of merged dataset and combined $p$-values of the genes in the hubs. 


\section{REFERENCES}

Ahmed, S. S., and Santosh, W. (2010). Metallomic profiling and linkage map analysis of early Parkinson's disease: a new insight to aluminum marker for the possible diagnosis. PLoS One 5:e11252. doi: 10.1371/journal.pone.0011252

Ahn, E., and Kang, H. (2018). Introduction to systematic review and meta-analysis. Korean J. Anesthesiol. 71, 103. doi: 10.4097/kjae.2018.71.2.103

Bocca, B., Alimonti, A., Senofonte, O., Pino, A., Violante, N., Petrucci, F., et al. (2006). Metal changes in CSF and peripheral compartments of parkinsonian patients. J. Neurol. Sci. 248, 23-30. doi: 10.1016/j.jns.2006.05.007

Bockaert, J., and Marin, P. (2015). mTOR in brain physiology and pathologies. Physiol. Rev. 95, 1157-1187. doi: 10.1152/physrev.00038.2014

Bosco, D., Plastino, M., Cristiano, D., Colica, C., Ermio, C., De Bartolo, M., et al. (2012). Dementia is associated with insulin resistance in patients with Parkinson's disease. J. Neurol. Sci. 315, 39-43.

Chi, J., Xie, Q., Jia, J., Liu, X., Sun, J., Deng, Y., et al. (2018). Integrated analysis and identification of novel biomarkers in Parkinson's disease. Front. Aging Neurosci. 10:178. doi: 10.3389/fnagi.2018.00178

Derkow, K., Rossling, R., Schipke, C., Kruger, C., Bauer, J., Fahling, M., et al. (2018). Distinct expression of the neurotoxic microRNA family Let-7 in the cerebrospinal fluid of patients with Alzheimer's disease. PLoS One 13:e0200602. doi: 10.1371/journal.pone. 0200602

Dias, V., Junn, E., and Mouradian, M. M. (2013). The role of oxidative stress in Parkinson's disease. J. Parkinsons Dis. 3, 461-491.

Dijkstra, A. A., Ingrassia, A., de Menezes, R. X., van Kesteren, R. E., Rozemuller, A. J., Heutink, P., et al. (2015). Evidence for immune response, axonal dysfunction and reduced endocytosis in the substantia nigra in early stage Parkinson's disease. PLoS One 10:e0128651. doi: 10.1371/journal.pone.012 8651

Du, K., Liu, M. Y., Zhong, X., and Wei, M. J. (2017). Decreased circulating Zinc levels in Parkinson's disease: a meta-analysis study. Sci. Rep. 7, 1-8.

Duarte, A. I., Moreira, P. I., and Oliveira, C. R. (2012). Insulin in central nervous system: more than just a peripheral hormone. J. Aging Res. 2012: 384017.

Duarte, R. R., Bachtel, N. D., Côtel, M. C., Lee, S. H., Selvackadunco, S., Watson, I. A., et al. (2019). The psychiatric risk gene NT5C2 regulates adenosine monophosphate-activated protein kinase signaling and protein translation in human neural progenitor cells. Biol. Psychiatry 86, 120-130. doi: 10.1016/j. biopsych.2019.03.977

Fiory, F., Perruolo, G., Cimmino, I., Cabaro, S., Pignalosa, F. C., Miele, C., et al. (2019). The relevance of insulin action in the dopaminergic system. Front. Neurosci. 13:868. doi: 10.3389/fnins.2019.00868

Forte, G., Bocca, B., Senofonte, O., Petrucci, F., Brusa, L., Stanzione, P., et al. (2004). Trace and major elements in whole blood, serum, cerebrospinal fluid and urine of patients with Parkinson's disease. J. Neural Trans. 111, 10311040.

Fukunaka, A., and Fujitani, Y. (2018). Role of zinc homeostasis in the pathogenesis of diabetes and obesity. Int. J. Mol. Sci. 19:476. doi: 10.3390/ijms19020476

Genoud, S., Senior, A. M., Hare, D. J., and Double, K. L. (2020). Meta-analysis of copper and iron in Parkinson's disease brain and biofluids. Mov. Disord. 35, 662-671. doi: $10.1002 / \mathrm{mds} .27947$

Hamed, M., Gladbach, Y., Möller, S., Fischer, S., Ernst, M., Struckmann, S., et al. (2018). A workflow for the integrative transcriptomic description of molecular pathology and the suggestion of normalizing compounds, exemplified by Parkinson's disease. Sci. Rep. 8, 1-12.

Iwakura, Y., and Nawa, H. (2013). ErbB1-4-dependent EGF/neuregulin signals and their cross talk in the central nervous system: pathological implications in schizophrenia and Parkinson's disease. Front. Cell. Neurosci. 7:4. doi: 10.3389/ fncel.2013.00004

Iwakura, Y., Piao, Y. S., Mizuno, M., Takei, N., Kakita, A., Takahashi, H., et al. (2005). Influences of dopaminergic lesion on epidermal growth factor-ErbB signals in Parkinson's disease and its model: neurotrophic implication in nigrostriatal neurons. J. Neurochem. 93, 974-983. doi: 10.1111/j.1471-4159. 2005.03073.x

Jin, X., Liu, M. Y., Zhang, D. F., Gao, H., and Wei, M. J. (2018). Elevated circulating magnesium levels in patients with Parkinson's disease: a meta-analysis. Neuropsychiatr. Dis. Treat. 14:3159. doi: 10.2147/ndt.s 186209
Kelly, J., Moyeed, R., Carroll, C., Albani, D., and Li, X. (2019). Gene expression meta-analysis of Parkinson's disease and its relationship with Alzheimer's disease. Mol. Brain 12, 1-10.

Khang, R., Park, C., and Shin, J. H. (2015). Dysregulation of parkin in the substantia nigra of db/db and high-fat diet mice. Neuroscience 294, 182-192. doi: 10.1016/ j.neuroscience.2015.03.017

Kleinridders, A., Cai, W., Cappellucci, L., Ghazarian, A., Collins, W. R., Vienberg, S. G., et al. (2015). Insulin resistance in brain alters dopamine turnover and causes behavioral disorders. Proc. Natl. Acad. Sci.U.S.A. 112, 3463-3468. doi: 10.1073/pnas.1500877112

Kostov, K. (2019). Effects of magnesium deficiency on mechanisms of insulin resistance in type 2 diabetes: focusing on the processes of insulin secretion and signaling. Int. J. Mol. Sci. 20:1351. doi: 10.3390/ijms20061351

Lang, A. E., and Lozano, A. M. (1998). Parkinson's disease. N. Engl. J. Med. 339, $1130-1143$.

Ledonne, A., Mango, D., Latagliata, E. C., Chiacchierini, G., Nobili, A., Nisticò, R., et al. (2018). Neuregulin 1/ErbB signalling modulates hippocampal mGluRIdependent LTD and object recognition memory. Pharmacol. Res. 130, 12-24. doi: 10.1016/j.phrs.2018.02.003

Ledonne, A., and Mercuri, N. B. (2020). On the modulatory roles of neuregulins/ErbB signaling on synaptic plasticity. Int. J. Mol. Sci. 21:275. doi: 10.3390/ijms 21010275

Lu, W., Guo, L., Jiang, T., Ni, S., and Zhao, Y. (2018). The effect of Mg $2 \mathrm{Zn}$ $0.5 \mathrm{Nd}$ alloy on the mTOR signalling pathway in L6 cells. Mol. Med. Rep. 18, 1885-1891.

Lund, C. V., Popkov, M., Magnenat, L., and Barbas, C. F. (2005). Zinc finger transcription factors designed for bispecificcoregulation of ErbB2 and ErbB3 receptors: insights into ErbB receptor biology. Mol. Cell. Biol. 25, 9082-9091. doi: $10.1128 / \mathrm{mcb} .25 .20 .9082-9091.2005$

Magrinelli, F., Picelli, A., Tocco, P., Federico, A., Roncari, L., Smania, N., et al. (2016). Pathophysiology of motor dysfunction in Parkinson's disease as the rationale for drug treatment and rehabilitation. Parkinsons Dis. 2016:9832839.

Moher, D., Shamseer, L., Clarke, M., Ghersi, D., Liberati, A., Petticrew, M., et al. (2015). Preferred reporting items for systematic review and meta-analysis protocols (PRISMA-P) 2015 statement. Syst. Rev. 4:1.

Moni, M. A., Rana, H. K., Islam, M. B., Ahmed, M. B., Hasan, M. A. M. Huq, F., et al. (2019). Detection of Parkinson's disease using blood and brain cells transcript profiles. bioRxiv[Preprint] 483016. doi: 10.1101/48 3016

Moreira, P. I., Cardoso, S. M., Santos, M. S., and Oliveira, C. R. (2006). The key role of mitochondria in Alzheimer's disease. J. Alzheimers Di. 9, 101-110.

Nepusz, T., Yu, H., and Paccanaro, A. (2012). Detecting overlapping protein complexes in protein-protein interaction networks. Nat. Methods 9:471. doi: 10.1038/nmeth.1938

Norouzi, S., Adulcikas, J., Sohal, S. S., and Myers, S. (2018). Zinc stimulates glucose oxidation and glycemic control by modulating the insulin signaling pathway in human and mouse skeletal muscle cell lines. PLoS One 13:e0191727. doi: 10.1371/journal.pone.0191727

Olivier, M., Asmis, R., Hawkins, G. A., Howard, T. D., and Cox, L. A. (2019). The need for multi-omics biomarker signatures in precision medicine. Int. J. Mol. Sci. 20:4781. doi: 10.3390/ijms20194781

Oyanagi, K., Kawakami, E., Kikuchi-Horie, K., Ohara, K., Ogata, K., Takahama, S., et al. (2006). Magnesium deficiency over generations in rats with special references to the pathogenesis of the parkinsonism-dementia complex and amyotrophic lateral sclerosis of Guam. Neuropathology 26, 115-128. doi: 10. $1111 / j .1440-1789.2006 .00672 . x$

Pagano, G., Niccolini, F., and Politis, M. (2016). Imaging in Parkinson's disease. Clin. Med. 16:371.

Pang, Y., Lin, S., Wright, C., Shen, J., Carter, K., Bhatt, A., et al. (2016). Intranasal insulin protects against substantia nigra dopaminergic neuronal loss and alleviates motor deficits induced by 6-OHDA in rats. Neuroscience 318, 157-165. doi: 10.1016/j.neuroscience.2016.01.020

Shen, X., Yang, H., Zhang, D., and Jiang, H. (2019). Iron concentration does not differ in blood but tends to decrease in cerebrospinal fluid in Parkinson's disease. Front. Neurosci. 13:939. doi: 10.3389/fnins.2019.00939

Su, L., Chen, S., Zheng, C., Wei, H., and Song, X. (2019). Meta-analysis of gene expression and identification of biological regulatory mechanisms in Alzheimer's Disease. Front. Neurosci. 13:633. doi: 10.3389/fnins.2019.00633 
Szewczyk, B., Pochwat, B., Rafało, A., Palucha-Poniewiera, A., Domin, H., and Nowak, G. (2015). Activation of mTOR dependent signaling pathway is a necessary mechanism of antidepressant-like activity of zinc. Neuropharmacology 99, 517-526. doi: 10.1016/j.neuropharm.2015.08. 026

Takeda, A. (2000). Movement of zinc and its functional significance in the brain. Brain Res. Rev. 34, 137-148. doi: 10.1016/s0165-0173(00)00 044-8

Tokiya, Y., and Toda, S. (1977). Application of atomic absorption spectroscopy for metal analysis in biological materials. Chem. Regul. Plants 26, 77-112. doi: 10.1007/BFb0051600

Wang, X., Kang, D. D., Shen, K., Song, C., Lu, S., Chang, L. C., et al. (2012). An R package suite for microarray meta-analysis in quality control, differentially expressed gene analysis and pathway enrichment detection. Bioinformatics 28, 2534-2536. doi: 10.1093/bioinformatics/ bts 485
Xu, Y., Liu, C., Chen, S., Ye, Y., Guo, M., Ren, Q., et al. (2014). Activation of AMPK and inactivation of Akt result in suppression of mTOR-mediated S6K1 and 4EBP1 pathways leading to neuronal cell death in in vitro models of Parkinson's disease. Cell. Signal. 26, 1680-1689. doi: 10.1016/j.cellsig.2014.04.009

Conflict of Interest: The authors declare that the research was conducted in the absence of any commercial or financial relationships that could be construed as a potential conflict of interest.

Copyright (C) 2021 Anirudhan, Angulo-Bejarano, Paramasivam, Manokaran, Kamath, Murugesan, Sharma and Ahmed. This is an open-access article distributed under the terms of the Creative Commons Attribution License (CC BY). The use, distribution or reproduction in other forums is permitted, provided the original author(s) and the copyright owner(s) are credited and that the original publication in this journal is cited, in accordance with accepted academic practice. No use, distribution or reproduction is permitted which does not comply with these terms. 